\title{
LIQUID DROP PARAMETERS FOR HOT NUCLEI
}

\author{
C. GUET ${ }^{1}$ \\ Ganil, B.P. 5027, F-14021 Caen, France
}

\author{
E. STRUMBERGER ${ }^{2}$ and M. BRACK ${ }^{3}$ \\ Universität Regensburg, D-8400 Regensburg, Fed. Rep. Germany
}

Received 3 February 1988

\begin{abstract}
Using the semi-classical extended Thomas-Fermi (ETF) density variational method, we derived self-consistently the liquid drop model (LDM) coefficients for the free energy of hot nuclear systems from a realistic effective interaction (Skyrme SkM*). We expand the temperature $(T)$ dependence of these coeffcients up to the second order in $T$ and test their application to the calculation of the fission barriers of the nuclei ${ }^{208} \mathrm{~Pb}$ and ${ }^{240} \mathrm{Pu}$.
\end{abstract}

The properties of hot nuclear systems formed in heavy ion collisions are under extensive experimental and theoretical study $[1,2]$. The equation of state of hot nuclear matter is also of great interest in astrophysics in connection with the formation of supernovae [3]. We shall use here a self-consistent mean field approach to calculate the binding energies of hot nuclei from an effective nucleon-nucleon interaction. This can be done in a very economical way in the density variational approach [4] using the recently developed extended Thomas-Fermi (ETF) density functionals for finite fermion systems at finite temperatures [5-7]. It was shown that this approach yields numerically the same results $[5,7]$ as the microscopical Hartree-Fock (HF) approach [8,9] for temperatures $T^{\# 1}$ larger than $2.5-3 \mathrm{MeV}$ where the shell effects have disappeared.

The purpose of this paper is to use the ETF density

Work supported in part by the French-German scientific exchange program PROCOPE.

1 Permanent address: Service de Physique, DRF, CENG, F38041 Grenoble, France.

2 Present address: Technische Universität München, D-8046 Garching, Fed. Rep. Germany.

3 Present address: Niels Bohr Institute, Blegdamsvej 17, DK-2 100 Copenhagen, Denmark.

\#1 We use MeV units for the temperature $T$, putting the Boltzmann constant $k=1$. functional approach to determine the coefficients in a liquid drop model (LDM) type expanson of the free energy of a hot nucleus. Such expansions have been very successful, mostly in the form of the droplet model [10], in describing the average binding energies of nuclei in their ground state $(T=0)$. They have recently also been applied in calculations of supernovae formation [11] and of the fragmentation of hot compound nuclei [12]. But in such applications, the temperature dependence of the LDM parameters is required as an input. This is precisely what can be derived systematically from ETF density variational calculations at finite temperature [4]. Here the free energy $F$ of the (spherical) nucleus is written in terms of its local density $\rho(r)$ as

$F=E-T S=\int f[\rho(r)] \mathrm{d}^{3} r=F[\rho]$,

where $E$ is the internal energy and $S$ the entropy. For the potential energy part of the functional $f[\rho]$ we use the Skyrme interaction $\mathrm{SkM}^{*}[4,13]$, for the kinetic energy and entropy densities the $T>0$ ETF functionals [6]. We refer to ref. [4] for the technical details of the leptodermous expansion of eq. (1) which follows from the assumption that the surface diffusivity of the local density $\rho(r)$ is much smaller than the radius $R$ at which the surface is located. The essence is that all the LDM or droplet model coeffi- 
cients can be related back to those obtained from a variational calculation with one-dimensional geometry, usually referred to as the (asymmetric) semiinfinite nuclear matter case (see also ref. [10]).

A word should be added here about the static treatment of a highly excited nucleus. Such a system is known not to be stable but to evaporate nucleons. However, at temperatures below about $4 \mathrm{MeV}$, the evaporation times are long enough $[14,15]$ so that the nucleus may be treated statically as a metastable system $[15,16]$, not unlike a superheated classical liquid drop [17]. The scenario is somewhat different in the astrophysical context of a collapsing star, where finite condensed nuclei may coexist with a gas of nucleons (and leptons) in local thermodynamical equilibrium $[3,11]$ under an external pressure. Such an equilibrium has been assumed also in recent $\mathrm{HF}$ calculations for finite nuclei at $T>0$ [14], where special attention was paid to the continuum contributions which are important in establishing the density of the surrounding nucleon gas. In refs. $[4,15]$ the leading LDM coefficients (i.e. the surface, curvature and constant terms of the symmetric case, see below) have been calculated for both situations: the equilibrium case with constant external pressure, and the metastable case corresponding to zero external pressure. It was found that for temperatures $T$ up to about 4 $\mathrm{MeV}$, the difference in the results could be neglected. (For a more detailed comparison of the two situatons, see ref. [18].) We have performed our present calculations in the metastable situation; the results, however, hold also for the equilibrium case as long as $T$ is not much larger than $4 \mathrm{MeV}$. In any case the present approach should not be used for temperatures higher than about $5 \mathrm{MeV}$ because of the onsetting of an instability of a nucleus against sudden break-up as indicated in different theoretical models $[12,14]$. (Note that no experimental evidence of compound nuclei above $5 \mathrm{MeV}$ has so far been reported.) In our present static approach where the ex- ternal gas phase is totally neglected [4] the instability shows up at $T=5.3 \mathrm{MeV}$ for ${ }^{208} \mathrm{~Pb}$. Beyond this limiting temperature, no minimum of the free energy $F$ can be found for the spherical nucleus. This temperature is lower than that of ref. [14] mainly because of the absence of an external pressure.

We use the following expansion of the total free energy $F$ of a spherical nucleus with nucleon number $A=N+Z$ :

$F=F_{\text {sym }}+F_{\text {as }}+F_{\text {Coul }}$.

The symmetric part is expanded in the usual LDM series in powers of $A^{-1 / 3}$ up to the constant term:

$F_{\text {sym }}(A)=a_{\mathrm{v}} A+a_{\mathrm{s}} A^{2 / 3}+a_{\mathrm{c}} A^{1 / 3}+a_{0}$.

The coefficients $a_{i}$ hereby are functions of the temperature $T$ which we approximate by

$a_{i}(T)=a_{i}(0)\left(1-x_{i} T^{2}\right)$.

The parameters $a_{i}(0)$ and $x_{i}$ are given in table 1 . It was shown recently [19] that the truncated expansion (3) reproduces the exact variational ETF energies of symmetric nuclei (without Coulomb interaction) at $T=0$ within less then $1 \mathrm{MeV}$ for $A>10$. (If three more terms are added, going up to the term proportional to $1 / A$, the accuracy is better than $0.1 \mathrm{MeV}$ [19].) We have confirmed in our present calculations that the quadratic approximation (4) leads to errors smaller than $1 \mathrm{MeV}$ in the total $F$ up to $T=4$ MeV.

It is a well-known result of the droplet model [10], confirmed also in the ETF variational calculations [4], that the asymmetry energy may not be expanded in a Taylor series in powers of $A^{-1 / 3}$. In accordance with the findings of refs. $[4,10]$, we thus write $F_{\text {as }}$ in the form

$F_{\text {as }}(A, I)=J A I^{2} /\left[1+(9 J / 4 Q) A^{-1 / 3}\right]$,

where

Table 1

Liquid drop and droplet model coefficients obtained from the SkM* interaction. The first row gives their values at zero temperature (in $\mathrm{MeV}$ ), the second row the coefficients $x_{i}$ [in $\mathrm{MeV}^{-2}$ according to eq. (4)].

\begin{tabular}{|c|c|c|c|c|c|c|c|c|c|}
\hline & $a_{\mathrm{v}}$ & $a_{\mathrm{s}}$ & $a_{\mathrm{c}}$ & $a_{0}$ & $J$ & $Q$ & $c_{1}$ & $c_{2}$ & $K_{\mathrm{inf}}$ \\
\hline$a_{i}(0)$ & -15.776 & 17.22 & 10.24 & -8.0 & 30.03 & 35.4 & 0.737 & -1.28 & 216.7 \\
\hline $10^{3} x_{i}$ & -3.36 & 5.53 & 5.43 & -12.3 & 1.04 & 12.8 & 0.763 & -13.2 & 5.99 \\
\hline
\end{tabular}


$I=(N-Z) / A$,

and $J$ and $Q$ are the volume asymmetry energy and the surface stiffness coefficient [10], respectively, which both are again fitted to the quadratic $T$ dependence (4).

Finally, the Coulomb energy - including the exchange part as usual in the Slater (or local density) approximation - is fitted by the following two parameter formula:

$F_{\text {Coul }}(A, Z)=c_{1} Z^{2} A^{-1 / 3}+c_{2} Z^{2} A^{-1}$.

We found that this simple formula reproduces the total Coulomb energies for nuclei with $6<A<250$ within less than $1 \mathrm{MeV}$ in the temperature range considered here. The parameters $c_{i}$, with their quadratic $T$ dependence according to eq. (4), are also contained in table 1.

We include in table 1 also the incompressibility parameter $K_{\text {inf }}$ of symmetric infinite nuclear matter and its temperature dependence in the quadratic approximation (4). This parameter, as well as the volume energy $a_{v}$ and the volume asymmetry energy $J$, are obtained analytically in terms of the Skyrme force parameters and the saturation density $\rho_{\text {inf }}$ which is equal to $0.1603 \mathrm{fm}^{-3}$ for the force $\mathrm{SkM}^{*}$ [4]. The parameters $a_{\mathrm{s}}$ and $a_{\mathrm{c}}$ are obtained from semi-infinite nuclear matter calculations in which the surface energy is minimized; the parameter $a_{c}$ contains also the so-called compression energy [10] and should be identified with the quantity $a_{c}^{*}$ of ref. [4]. The first three terms of eq. (3) therefore are those of a strict asymptotic expansion in the large $A$ limit (see ref. [19]). The constant term $a_{0}$ is adjusted such [19] that eq. (3) reproduces the variational ETF free energies within less than $1 \mathrm{MeV}$ for $A>10$. According to the results of refs. [4-6], the total free energies eq. (2) should also reproduce the exact HF results for temperatures $T$ above $2.5-3 \mathrm{MeV}$ where there are no more shell effects [7-9]. Even for low temperatures, the parametrisation by eqs. (2)-(7) may be used, together with temperature dependent shell corrections, to yield an excellent approximation to the microscopic HF free energies by exploiting the Strutinsky energy theorem at $T>0[20]$.

The coefficients $K_{\mathrm{inf}}, a_{\mathrm{s}}, a_{\mathrm{c}}$ and $a_{0}$ all go to zero at the critical temperature $T_{\mathrm{c}}$ [4] which (for the SkM* force) equals $14.6 \mathrm{MeV}$ in the equilibrium case. The quadratic approximation to the temperature dependence of the first three parameters is excellent and illustrated in fig. 1, even up to temperatures of about 8-9 MeV (see ref. [4] for $a_{0}$ which is quadratic only up to $T=4 \mathrm{MeV}$, and for the metastable situation where the above coefficients vanish at $T=11.6 \mathrm{MeV}$ ).

It is straightforward to calculate the entropy $S$ of a finite nucleus from the above results by the canonical relation $S=-\mathrm{d} F / \mathrm{d} T$, and from it the level density parameter $a_{\text {lev }}$ by the Fermi gas relation $S=2 a_{\mathrm{lev}} T$ which was found to be well fulfilled for $2<T<4 \mathrm{MeV}$ also in the ETF model [4,5] and the $T>0 \mathrm{HF}$ calculations [8] (note that at large temperatures the mean field estimate of $a_{\mathrm{lev}}$ obtained here is expected to be the correct one $[21,22]$ ).

As an application of our "hot" LDM coefficients, we finally want to discuss the temperature dependence of fission barriers of heavy nuclei. In ref. [4] some preliminary results of fission barrier calculations at $T>0$ have already been presented for ${ }^{240} \mathrm{Pu}$. We have repeated these calculations, incorporating now also the correct temperature dependent fourthorder gradient corrections of the kinetic free energy density [6].

In fig. 2 we show the temperature dependence of the height of the fission barrier of ${ }^{208} \mathrm{~Pb}$ and ${ }^{240} \mathrm{Pu}$ (see ref. [4] for the parameterisation of the deformed nuclear shapes ). Note that the SkM* Skyrme force was specifically chosen to reproduce the empirical LDM fission barrier of ${ }^{240} \mathrm{Pu}[4,13]$. The semi-

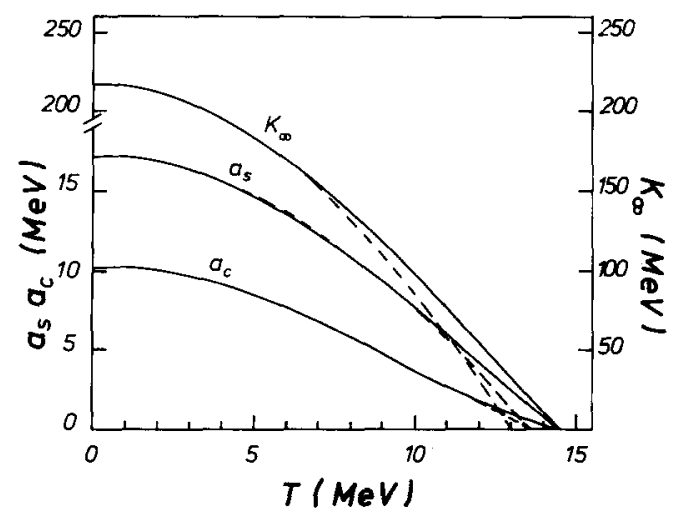

Fig. 1. Incompressibility $K_{\infty}$, surface energy $a_{\mathrm{s}}$ and curvature energy $a_{\mathrm{c}}$ obtained from the SkM* force, versus temperature. Solid lines: equilibrium between gas and liquid phase assumed. Dashed lines: quadratic approximation eq. (4) with parameters in table 1. 


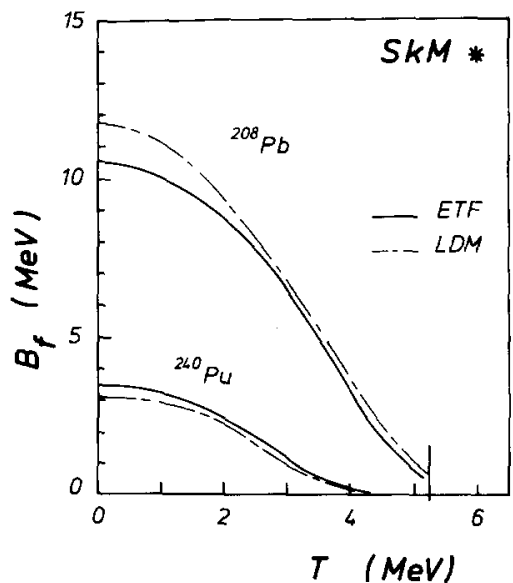

Fig. 2. Fission barrier heights obtained by semi-classical ETF variational calculations (for details see ref. [4]) with the Skyrme force $\mathrm{SkM}^{*}$ (solid lines). Dash-dotted lines: LDM barriers, calculation is described in the text. The cut-off of the ${ }^{208} \mathrm{~Pb}$ curves corresponds to the temperatures $T=5.3 \mathrm{MeV}$ above which no variational minimum of the free energy $F$ is found.

classical fission barrier of ${ }^{208} \mathrm{~Pb}$ at $T=0$ is found to be slightly lower (by about 1-2 MeV) than the empirical LDM one [23]. It is now a very crucial test to see whether these results can be fitted by the simple liquid drop model. In this model the deformation energy of the nucleus is written

$F_{\mathrm{LD}}($ def $)=F_{\mathrm{s}}(0) B_{\mathrm{s}}+E_{\text {Coul }}(0) B_{\text {Coul }}$,

where $F_{\mathrm{s}}(0)$ and $E_{\mathrm{Coul}}(0)$ are the surface and Coulomb energy of the equivalent sharp-edged liquid drop, respectively. The coefficients $B_{\mathrm{s}}$ and $B_{\text {Coul }}$ contain the deformation dependence [10]. Since the total nuclear free energy in eq. (2) contains more than surface energy terms we define an effective surface free energy of the spherical nucleus under consideration, which incorporates curvature, higher order and asymmetry effects (see also ref. [24]):

$F_{\mathrm{s}}^{\mathrm{eff}}=a_{\mathrm{s}} A^{2 / 3}+a_{\mathrm{c}} A^{1 / 3}+F_{\mathrm{as}}(A, I)-J A I^{2}$,

with the parameters given in table 1 . The deformation dependence of the curvature energy is, in fact, not too different from that of the surface energy for typical saddlepoint shapes of actinide nuclei [25]. The equivalent sharp proton radius appearing in the Coulomb energy is well approximated for heavy nuclei by the quadratic relation

$R_{\mathrm{p}}^{\mathrm{eq}}(T)=r_{0} A^{1 / 3}\left[1+x_{\mathrm{R}} T^{2}\right]$, with $r_{0}=1.15 \mathrm{fm}$ and $x_{\mathrm{R}}=0.710^{-3} \mathrm{MeV}^{-2}$. The fission barriers found in this way are shown by the dashed lines in fig. 2. Note that the discrepancy of 1.2 $\mathrm{MeV}$ observed at $T=0$ in ${ }^{208} \mathrm{~Pb}$ corresponds to an error of less than 2 parts in 1000 of either the surface energy or the Coulomb energy at the saddle-point. The accuracy required to obtain the correct semi-classical barrier height at $T=0$ therefore is clearly beyond what can be achieved in a simple sharp-edged liquid drop picture. Note however, that the LDM curve in fig. 2 correctly reproduces the $T$ dependence of the ETF barrier height.

For practical use, we have checked that the wellknown LDM formula [10] for the barrier height:

$B_{\mathrm{f}}=0.83 F_{\mathrm{s}}^{\mathrm{eff}}(T)[1-x(T)]^{3}$ (in $\left.\mathrm{MeV}\right)$,

reproduces very well the LDM curves in fig. 2 up to $T=4 \mathrm{MeV}$. The temperature-dependent fissility parameter $x(T)$ is then defined by

$$
\begin{aligned}
x(T) & =\left[1 / 2 F_{\mathrm{s}}^{\mathrm{ef}}(T)\right] \frac{3}{5} Z^{2} e^{2} / R_{\mathrm{p}}^{\mathrm{eq}}(T) \\
& =x(0)\left(1+x_{\mathrm{f}} T^{2}\right), \\
x_{\mathrm{f}} & =7.1 \times 10^{-3} \mathrm{MeV}^{-2} .
\end{aligned}
$$

To summarize we want to stress that the aim of this paper was not to give a mass formula with all the sophistications necessary of the droplet model, but rather to give a useful parameterisation of the $T$-dependence of the leading LDM and droplet model coefficients, derived in a self-consistent way from a realistic effective Skyrme interaction. We have shown that also the temperature dependence of fission barriers can be correctly reproduced in terms of these coefficients and parametrisations. For the calculation of the total average energies and fission barriers at $T=0$, we still advocate the use of the variational ETF model which incorporates all surface diffuseness effects and interactions of Coulomb and nuclear parts in the energy which we ignored in the above simple LDM expressions (see also conclusions of ref. [4]).

The authors are grateful to Kim Sneppen for his interest in this work and for stimulating discussions. Two of us (C.G. and M.B.) acknowledge the hospitality of their mutual institutions and that of the Niels Bohr Institute in Copenhagen, where this work was completed. 


\section{References}

[1] See e.g. Intern. Conf. on Heavy ion nuclear collisions in the Fermi energy domain HICOFED (Caen, 1986), J. Phys. (Paris) C 4 (1986) 1.

[2] C.K. Gelbke, ed.,Proc. Symp. on Central collisions and fragmentation processes (Denver, 1987), Nucl. Phys. A 471 (1987) 1c-451c.

[3] E. Baron, H.A. Bethe, G.E. Brown, J. Cooperstein and S. Kahana, preprint BNL 39814, and references therein.

[4] M. Brack, C. Guet and H.-B. Haakansson, Phys. Rep. 123 (1985) 263.

[5] M. Brack, Phys. Rev. Lett. 53 (1984) 119; 54 (1985) 851.

[6] J. Bartel, M. Brack and M. Durand, Nucl. Phys. A 445 (1985) 263.

[7] M. Brack and J. Bartel, 4th Intern. Conf. on Recent progress in many-body theories (San Francisco, 1985), eds. P. Siemens and R. Smith (Springer, Berlin, 1987), to be published.

[8] M. Brack and P. Quentin, Phys. Lett. B 52 (1974) 159; Phys. Scr. A 10 (1974) 163.

[9] G. Sauer, H. Chandra and U. Mosel, Nucl. Phys. A 264 (1976) 221 , and references therein.

[10] W.D. Myers and W.J. Swiatecki, Ann. Phys. (NY) 55 (1969) 395.
[11] C.J. Pethick, D.G. Ravenhall and J.M. Lattimer, Phys. Lett. B 128 (1983) 137.

[12] J. Bondorf et al., Nucl. Phys. A 443 (1985) 321; A 444 (1985) 460

[13] J. Bartel, P. Quentin, M. Brack, C. Guet and H.-B. Haakansson, Nucl. Phys. A 386 (1982) 79.

[14] P. Bonche, S. Levit and D. Vautherin, Nucl. Phys. A 436 (1985) 265, and references therein.

[15] M. Brack, Phase space approach to nuclear dynamics, eds. M. Di Toro et al. (World Scientific, Singapore, 1986) p. 417.

[16] W. Stocker and J. Burzlaff, Nucl. Phys. A 202 (1973) 265.

[17] See e.g. H.N.V. Temperley, Proc. Phys. Soc. 59 (1947) 199.

[18] A. Blin, M. Brack and E. Strumberger, to be published.

[19] W. Stocker, P. Gleissl and M. Brack, Nucl. Phys. A 471 (1987) 501

[20] M. Brack and P. Quentin, Nucl. Phys. A 361 (1981) 35.

[21] A. Lejeune, P. Grange, M. Martzolff and J. Cugnon, Nucl. Phys. A 453 (1986) 189.

[22] R.W. Hasse and P. Schuck, Phys. Lett. B 179 (1986) 313.

[23] A. Sierk and J.R. Nix, private communication.

[24] J. Bartel and P. Quentin, Phys. Lett. B 152 (1985) 29

[25] M. Brack et al., Fourth Intern. Conf. on Nuclei far from stability (Hölsingër, 1981), CERN 81-01 (Geneva) p. 65 . 